\title{
Prediction of tool-wear in turning of medical grade cobalt chromium molybdenum alloy (ASTM F75) using non-parametric Bayesian models
}

\author{
Damien McParland · Szymon Baron · Sarah \\ O'Rourke · Denis Dowling · Eamonn Ahearne . \\ Andrew Parnell
}

Received: date / Accepted: date

\begin{abstract}
We present a novel approach to estimating the effect of control parameters on tool wear rates and related changes in the three force components in turning of medical grade Co-Cr-Mo (ASTM F75) alloy. Co-Cr-Mo is known to be a difficult to cut material which, due to a combination of mechanical and physical properties, is used for the critical structural components of implantable medical prosthetics. We run a designed experiment which enables us to estimate tool wear from feed rate and cutting speed, and constrain them using a Bayesian hierarchical Gaussian Process model which enables prediction of tool wear rates for untried experimental settings. However, the predicted tool wear rates are non-linear and, using our models, we can identify experimental settings which optimise the life of the tool. This approach has potential in the future for realtime application of data analytics to machining processes.
\end{abstract}

Keywords Cobalt Chromium Alloys · Orthogonal Cutting · Forces in Cutting · Gaussian Process · Tool Life Optimisation

Damien McParland

School of Mathematics and Statistics, University College Dublin, Ireland.

E-mail: damien.mcparland@ucd.ie

Szymon Baron

School of Mechanical and Materials Engineering, University College Dublin, Ireland.

Sarah O'Rourke

Central Statistics Office of Ireland, Dublin, Ireland.

Eamonn Ahearne

School of Mechanical and Materials Engineering, University College Dublin, Ireland.

Denis Dowling

School of Mechanical and Materials Engineering, University College Dublin, Ireland.

Andrew Parnell

School of Mathematics and Statistics, University College Dublin, Ireland.

Insight Centre for Data Analytics, University College Dublin, Ireland. 


\section{Introduction}

Technological advances in high-end manufacturing and ever stringent international standards drive the growth in demand for complex components and high precision tolerances. Automotive, aeronautical and medical device industries often rely on a business platform that involves the use of CAD/CAM technologies and multi-axis computer numerically controlled machine tools. The overall capability of this process chain is often constrained by the core cutting process and the fundamental mechanisms that result in thermal error, deflection and tool-wear (Altintas, 2012; Shaw, 2005). The constraints imposed by the underlying fundamental mechanisms of cutting are further exacerbated when machining difficult to cut (DTC) materials such as cobalt, nickel or titanium based superalloys (Williams, 2008).

Co-Cr-Mo alloys are well established as materials for medical devices and orthopaedic implants in particular (Williams, 2008; Al Jabbari, 2014). High mechanical strength and wear resistance make them suitable even for applications in metal-on-metal bearing components while biocompatibility allows for long-term incorporation in the human musculoskeletal system (Williams, 2008). In developed economies, factors such as increased physical activity, rising rates of obesity and increased life expectancy are considered as the main drivers of growth in demand for knee and hip arthroplasties (Tozzi, 2015; Centers for Disease Control and Prevention, 2016). Given the risks associated with revision surgeries, the Co-Cr-Mo components are expected to perform to the required standard for up to 20 years (Buechel $\mathrm{Sr}$ et al, 2001). This imposes the need for high precision tolerances and surface finish while growth in demand presents challenges for high levels of process throughput.

Tool-wear, defined as gradual loss of the tool material from the areas of contact with the workpiece, directly affects these process measures (Altintas, 2012; Shaw, 2005). Despite the importance of the application, market size and future challenges, there are very few publications on machining of Co-Cr-Mo alloys. Furthermore any predictive models of toollife are often unique for particular tool-material-cutting fluid-machine configuration (Shaw, 2005; Halpin, 2012). More recently it has been advocated that the era of Industry 4.0 and/or digital manufacturing (DM) may contribute to the deconvolution of inherent complexity of processes (Ahearne et al, 2016). This may be achieved through incorporation of disruptive technologies such as new smart sensors, the internet of things, advanced data analytics and cloud computing (Ahearne et al, 2016; Byrne et al, 2016). The principle is to follow a systematic progression in 'levels of intelligence' as shown on Figure 1 below. Incorporation of disruptive technologies into machining processes requires input from sensing equipment, signal processing and decision making support systems.

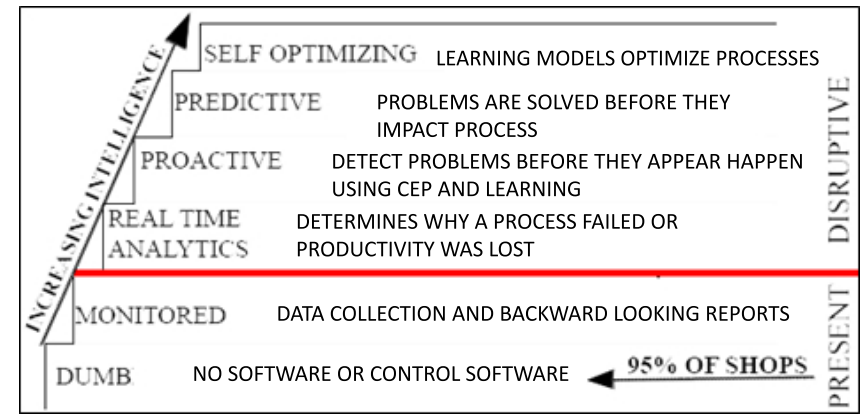

Fig. 1: Roadmap for machining process control. 
Our previous work detailed the properties of Co-Cr-Mo alloys and indicated that these alloys should be classified as DTC materials (Baron et al, 2015). This paper now presents an approach that utilises non-parametric Bayesian hierarchical modelling to determine the rates of tool wear and estimate the cutting lengths at which changes in these rates occur, such as the length of cut when tool failure occurs. For each set of control parameters, the progression of tool wear is modelled against the profile of the cutting forces recorded by a piezoelectric dynamometer. As part of our suite of output, we present response surface plots and estimated tool life values.

The paper proceeds as follows: Section 2 provides an overview of other research done in these areas and in Section 3 the experimental design and statistical algorithms used to analyse the collected data are explained in detail. In Section 4 the results of applying these methods to the Co-Cr-Mo ASTMF75 cutting data are presented. Finally, the paper finishes with a discussion in Section 5.

\section{Previous Work}

Shaw (2005) defines metal cutting as the removal of a macroscopic chip by a tool with a defined cutting edge. Turning, milling and drilling are common cutting operations. In turning, "a single point tool removes unwanted material to produce a surface of revolution" (Shaw, 2005). The multi-physics mechanisms that take place during this process may be explained with reference to simplified orthogonal cutting as proposed by Merchant and enhanced by his model of the cutting mechanics (Merchant, 1945; Markopoulos, 2013). In the primary deformation zone as shown in Figure 2 the workpiece material is subject to extreme strain rates of $2.0 \cdot 10^{4} \mathrm{~s}^{-1}$ and temperatures of $1200^{\circ} \mathrm{C}$ (Jaspers and Dautzenberg, 2002) in machining of Inconel 718 (Dudzinski et al, 2004).

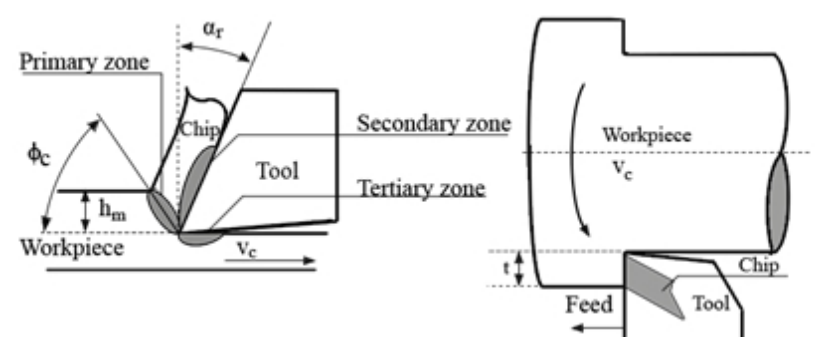

Fig. 2: Schematic typical wear patterns

The primary source of heat is the deformation in the primary shear zones while the secondary and tertiary sources of heat are primarily due to friction at (a) the tool-chip interface in the secondary shear zone and (b) the tool-workpiece interface at the tertiary shear zone (Abukhshim et al, 2006). The majority of the heat generated in this process is carried away with the chip (Shaw, 2005). In order to form the chip, the cutting tool must have a hardness higher than the hardness of the workpiece material. In addition, the cutting tool material must possess a combination of: (i) high-temperature hardness and chemical stability (ii) abrasive wear resistance (iii) resistance to brittle fracture. Tool wear is defined as a gradual loss of the tool material in the tool-workpiece contact zones (Klocke and Krieg, 1999). When the quality of the tool cutting edge is lost because of wear or breakage, the tool must 
be replaced. Cutting tools experience various modes of wear such as: flank, notch, crater wear and edge chipping (Altintas, 2012). These modes result from a combination of wear mechanisms, some of which include: adhesion, abrasion, oxidation, diffusion and fracture wear. Figure 3 shows some examples of this type of wear.

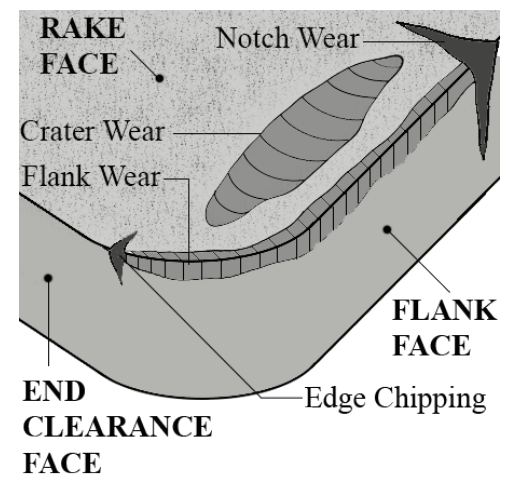

Fig. 3: Schematic of 2D chip formation (left) and a single point turning (right).

The tool wear rates depend on a range of fixed process and control parameters. Taylor's tool life equation is an example of a simple model describing the relationship between tool life and the cutting conditions (Altintas, 2012):

$$
v_{c} T^{n}=C
$$

where $v_{c}$ is the cutting speed, $T$ is the tool life, $n$ and $C$ are experimentally determined material constants (Shaw, 2005). The cutting speed and the chip thickness are thus indicated to be the main cutting conditions that affect the tool wear rates. A significant amount of research has focused on optimisation of control parameters given combinations of the workpiece material, machine and cutting tool-characteristics. The process constraints and costs associated with the tool-wear are further aggravated when machining difficult to cut alloys (Shaw, 2005). Machinability of an alloy is subject to its characteristics, where titanium, nickel and cobalt based alloys are widely regarded as DTC. In previous work (Baron et al, 2015) we compared Co-Cr-Mo alloy with Ti-6Al-4V and subsequently classified it as DTC. Cobalt and nickel based alloys contain highly abrasive, carbide particles generating abrasive wear (Shaw, 2005). The relatively low thermal conductivity of these alloys and high work hardening rates results in heat generation, resulting in temperatures of up to $1200^{\circ} \mathrm{C}$ and high magnitudes of stresses at the tool-workpiece interface (Altintas, 2012; Settineri et al, 2008). These conditions promote oxidation and diffusion of tool material. Common countermeasures applied to carbide tooling are PVC or CVD coatings of TiN or TiAlN. More recent efforts also include (i) rake and/or flank face structuring (ii) cryogenic machining (Altintas, 2012) (iii) nanostructure and superlattice PVD single and multi-coating layer coatings (Uhlmann et al, 2009).

The advances outlined above focus on either prolonging the tool-life or increasing the material removal rates but do not advance in the 'levels of intelligence' (Figure 1). As the tool-life is often application specific, the majority of production chains continue to utilise machining-time tool change criteria (Altintas, 2012). Measurement of the cutting force is an 
example of indirect, in-process measurement while periodic tool edge inspection is an example of direct, intermittent tool condition monitoring (TCM) strategy (Byrne et al, 2016). Incorporation of a machining process TCM system for an unattended manufacturing process relies on sensors that measure a range of in-process variables influenced by the condition of the cutting tool. The output of the sensors is subject to analogue and/or digital signal conditioning followed by signal processing in order to generate parameters that are correlated with the state of the cutting tool (Teti et al, 2010). These parameters are an input to a decision making support system for final interpretation. The output from the decision making system can be communicated to the machine tool operator or fed to the CNC controller for execution (Teti et al, 2010).

Models to predict the tool-wear in the time-domain include auto regressive, moving average and auto-regressive moving average (Teti et al, 2010; Dimla and Lister, 2000; Abouelatta and Madl, 2001). Advancement in 'levels of intelligence' and incorporation of tool TCM system in unattended manufacturing process requires effective decision making support structure. The cognitive systems most commonly employed for this purpose include neural networks, fuzzy logic, generic algorithms and hybrid systems (Byrne et al, 1995; Teti et al, 2010).

In this paper, we employ a Bayesian approach to statistical modelling of tool wear. The Bayesian approach admits the incorporation of expert prior knowledge into the estimation of model parameters. This is implemented by the specification of prior distributions. A comprehensive explanation of Bayesian data analysis is provided in Gelman et al (2003). As part of our modelling approach, we use non-parametric Bayesian models known as Gaussian processes. These allow us to specify an infinite dimensional Gaussian distribution which can flexibly adapt to observed data. This technique is utilised here to link model parameters under different experimental conditions by borrowing strength between experiments. Posterior predictive distributions of the Gaussian Process are used to estimate the model parameters for experimental conditions not used in the study.

In Bayesian analysis, prior distributions are combined with the experimental data to produce a posterior distribution of the parameters given the data. It is often difficult to perform inference based on this distribution directly since the distribution itself is often intractable. Instead, inference is done via a Markov chain Monte Carlo (MCMC) algorithm that draws samples from the posterior distribution. More information on MCMC algorithms can be found in Brooks et al (2011). Much development has taken place in MCMC algorithms over the last 20 years. One recent major development was Hamiltonian Monte Carlo (HMC) algorithms (Neal et al, 2011; Girolami and Calderhead, 2011; Hoffman and Gelman, 2014). These algorithms make intelligent proposals based on first order gradient information. We use the HMC algorithm implemented in the Stan software (Carpenter et al, 2015) which is interfaced with $\mathrm{R}$ using the rstan package.

\section{Methods}

\subsection{Experimental Plan and Design}

We perform single point turning tool-wear experiments to examine the influence of the process control parameters. We vary cutting speed $v_{c}(\mathrm{~m} / \mathrm{min})$ and feed-rate $f(\mathrm{~mm} / \mathrm{rev})$, and evaluate their effects on the tool-wear rates and resultant forces in the tangential force, $F_{t}$, feed force, $F_{f}$, and axial (passive) force, $F_{p}$, directions (Figure 4 ). It should be noted here that the feed rate $(\mu \mathrm{m} / \mathrm{rev})$ in turning is comparable to the more basic undeformed chip 
thickness referred to in analysis of cutting processes. The experimental parameters were based on on our previous research into orthogonal cutting of Co-Cr-Mo alloy ASTM F1537 standard reported in Baron and Ahearne (2015).

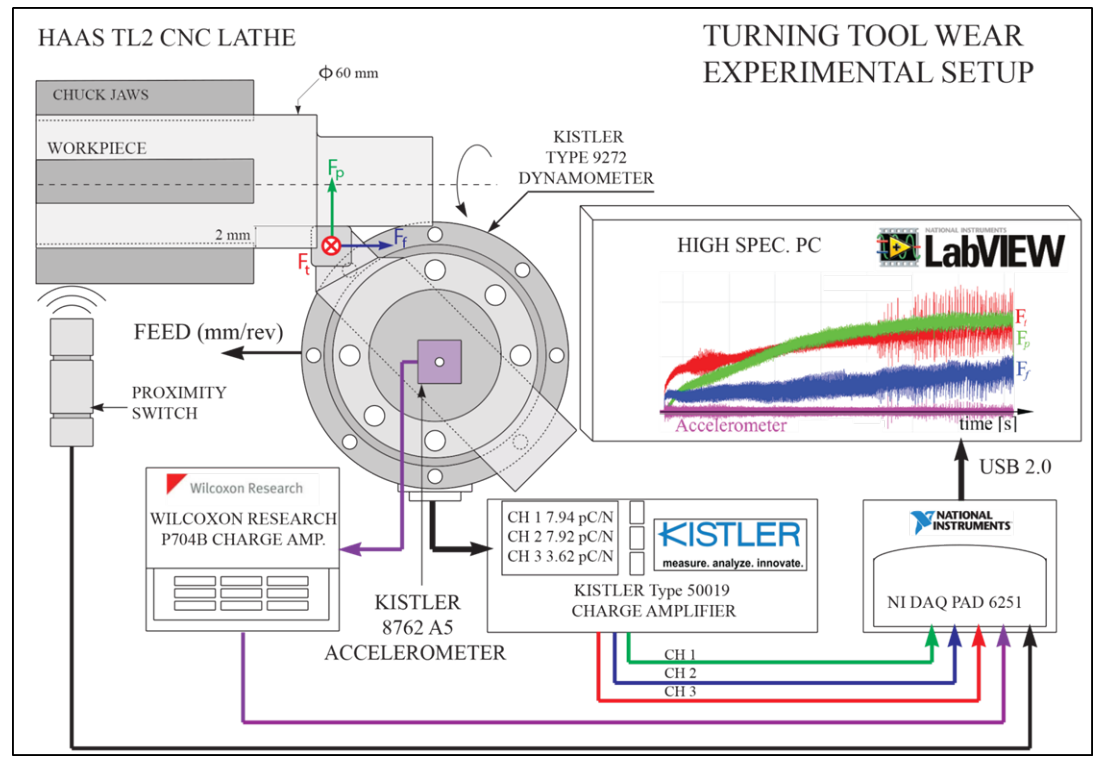

Fig. 4: Configuration of the tool-wear experimental setup.

A full factorial randomised design over the feasible ranges for cutting speed and feedrate was set-up using a Sobol sequence (Sobol, 1967). This sequence seeks to minimise any gaps or holes in the multidimensional space whilst simultaneously minimising any holes in projections of the sequence into a lower number of dimensions. The sequence initially contains points that cover the range of settings in a coarse manner and, as the sequence progresses, points are added to obtain a finer degree of coverage. This behaviour supports progressive augmentation of test settings (Azmin and Stobart, 2015) thus allowing for the maximum number of tests in the time available to complete the experiment. Figure 5 shows the first 21 prioritised test settings that were used in the experiment plus the following 9 test settings that could have been used to augment the data if time had allowed. The tests are run at the settings corresponding to the larger points first, moving to the smaller points afterwards. It is clear that the sequence provides an initially coarse coverage that becomes progressively finer. This design enables estimation of non-linear effects and interactions between control variables, whilst maximising the time value of the experiment.

\subsection{Experimental Procedures}

The tests were carried out on the HAAS TL2 CNC lathe with an $8.9 \mathrm{~kW}$ spindle motor rated for maximum speed of 2000 RPM and driving a $208 \mathrm{~mm}$ capacity chuck. The forces, $F_{t}, F_{p}$, and $F_{f}$ were recorded using a force measuring system comprised of Kistler Type 9272 dynamometer, a Kistler Type 5019 charge amplifier. The dynamometer was secured 


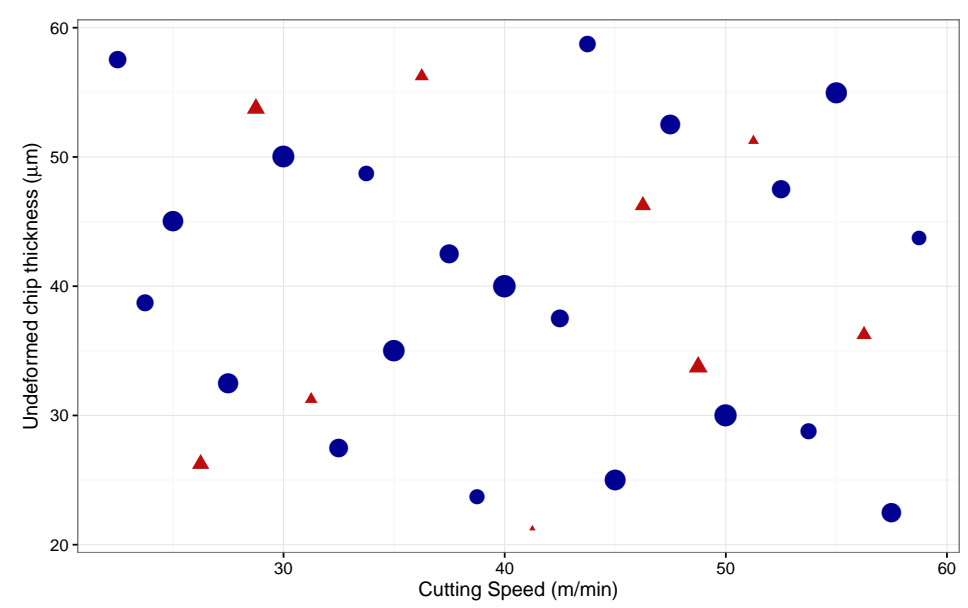

Fig. 5: Experimental design layout following a Sobol sequence. The larger points denote experimental settings considered first while settings tested later are smaller. The 21 blue discs denote settings that were actually used while the 9 red triangles denote the following points in the sequence for which there was not time to conduct the experiments. It can be seen that the sequence provides initially coarse coverage that gets progressively finer.

on the machine tool using a rigid fixture system. The fixture-dynamometer setup was analysed using FEA for stiffness in the horizontal and the vertical planes (found to be 148 and $181 \mathrm{kNmm}^{-1}$ respectively). A modal analysis of this assembly, assuming infinite tool-base rigidity showed lowest modal frequency to be $3.6 \mathrm{kHz}$, well above the in-plane dynamometer natural frequency of $1.5 \mathrm{kHz}$. Prior to the experiment, the force measuring system was cross calibrated off the machine on a Hounsfield tensometer. It was found to conform within 5\% of the Hounsfield measurement over the range from 0 to $4 \mathrm{kN}$. Tool vibration was monitored using a Kistler 8762A5 accelerometer with Wilcoxon Research P704B charge amplifier. A proximity sensor was installed on the machine to allow for accurate determination of the number of rotations and the rotational speed of the chuck. It therefore allowed for verification of the process control parameters. The output of the proximity switch, force, and vibration measuring system was recorded using NI DAQ PAD 6051 and LabView software with the sampling rate set to $150 \mathrm{kHz}$. The schematic of the experimental setup is shown in Digure 4. The force data was post-processed and analysed using NI DiaDem2014 software which included a low-pass digital filtering of $1.5 \mathrm{kHz}$ in order to remove any influence of dynamometer resonant frequency of $1.5 \mathrm{kHz}$ Kistler (2016). Modal analysis was used to determine the work offset length to ensure stability. As a result a round Co-Cr-Mo ASTM F75 bar of $61 \mathrm{~mm}$ diameter was secured in the chuck such that the work offset, measured from the chuck, was $120 \mathrm{~mm}$. The chemical composition of the workpiece is given in Table 1 .

Table 1: Chemical composition requirements of alloy conforming to ASTM F75 standard.

\begin{tabular}{lcccccccc}
\hline Material & $\mathrm{Cr}$ & $\mathrm{Mo}$ & $\mathrm{Ni}$ & $\mathrm{Fe}$ & $\mathrm{C}$ & $\mathrm{Si}$ & $\mathrm{Mn}$ & $\mathrm{Co}$ \\
\hline ASTM F75 (ASTM, 2016) & $27-30$ & $5-7$ & $<0.5$ & $\leq 0.75$ & $<0.14$ & $<1$ & $<1$ & Bal. \\
\hline
\end{tabular}


The workpiece material nominal properties, listed in Table 3, were validated in part by hardness measurements taken at intervals in the radial and axial directions where the mean measured hardness is given in Table 2 .

Table 2: Material nominal properties of an alloy conforming to ASTM F75 standard.

\begin{tabular}{lcc}
\hline & Units & ASTM F75 (Kaiser, 2013) \\
\hline Tensile Strength & $\mathrm{MPa}$ & 920 \\
0.2\% Proof Stress & $\mathrm{MPa}$ & 527 \\
Elongation & $\%$ & 16.5 \\
Young's Modulus & $\mathrm{GPa}$ & 216 \\
Hardness & $\mathrm{HRC}$ & 34 \\
Density $(\rho)$ & $\mathrm{Kg} \cdot \mathrm{m}^{-3}$ & 8,300 \\
Thermal Diffusivity & $\mathrm{m}^{2} \cdot \mathrm{s}^{-1}$ & $3 \times 10^{-6}$ \\
\hline
\end{tabular}

Table 3: Detailed list of fixed experimental parameters.

\begin{tabular}{lll}
\hline Test Parameter & Units: & Value: \\
\hline Work Material & & \\
\hline Material properties: & & See Table 2 \\
Stock Shape Dimensions & $\mathrm{mm}$ & ASTM F75 $\emptyset$ 62 Round bar \\
Measured Hardness & HV(HRC) & $322(34)$ for ASTM F75 \\
Depth of cut & $\mathrm{mm}$ & 1.9 \\
\hline Tool Insert & & \\
\hline Insert Code: & N/A & SCMW 120408 H13A \\
Rake Angle: & $\circ$ & 0 \\
Relief Angle: & $\circ$ & 7 \\
Nose Radius & $m m$ & 0.8 \\
Cutting Edge Radius, $r_{\beta}:$ & $\mu \mathrm{m}$ & 14 \\
\hline Cutting Fluid & & \\
\hline Cutting Fluid Type: & N/A & FUSCH ECOCOOL ULTRALIFE A \\
Cutting Fluid Flow: & $1 . \mathrm{min}^{-1}$ & 2.5 \\
Concentration: & $\%$ & 8.50 \\
\hline
\end{tabular}

The test procedure involved running an outside-diameter turning operation with the depth of cut equal to $1.9 \mathrm{~mm}$. The tool moved in the axial direction at a set feed-rate $(\mu \mathrm{m} / \mathrm{rev})$ with the chuck rotating at fixed rpm such that the pre-determined cutting speed was achieved. The wear scar on the rake and the flank face of the insert was first photographed and measured using a Keyence VHX-2000 optical microscope at intervals corresponding to $\approx 20 \mathrm{~m}$ length of the spiral of removed material. The criterion for determining the tool life $(\mathrm{m})$, the value of cutting length when the fool failure occurs, was either a visible increase in the variance of $F_{t}$ or a corresponding flank wear value greater than $200 \mu \mathrm{m}$ in cases where a clear change in the variance of $F_{t}$ was not visible. In general, there was a visible change in $F_{t}$ for higher cutting speeds while for lower cutting speeds the tool-wear was more gradual. It must be noted that the flank wear criterion could be exceeded before 
the onset of the force variance criterion. In the case of gradual tool wear, the length of cut corresponding to the closest value of $200 \mu \mathrm{m}$ flank wear was selected as the value of tool life.

\subsection{Statistical Methods}

We use a Bayesian Hierarchical Model (Gelman and Hill, 2006) to simultaneously learn the tool wear rates in each force direction whilst constraining them in an $\mathbb{R}^{2}$ random field according to their cutting speeds and feed rate. The Bayesian approach allows us to borrow strength between experiments by using informative prior distributions on the latent parameters of interest, and thus to improve the precision of our estimates. The top level of our model (the likelihood) is a simple linear regression of force against length of cut for each experiment. At the second level the latent slopes (representing e.g. the feed force) are given a non-parametric prior which regularises their values and allows us to predict feed force for experiments not yet run.

We start by defining the pair $\left(F_{i j}, L_{i j}\right)$ to be respectively the force (either $F_{t}, F_{f}$ or $F_{p}$ ) and length measurements for experiment $i(i=1, \ldots, K$ experiments) and measurement $j$ $\left(j=1, \ldots, N_{i}\right.$ measurements for experiment $\left.i\right)$. The top level of the model is then:

$$
F_{i j}=\alpha_{i}+\beta_{i} L_{i j}+\varepsilon_{i j}
$$

where $\alpha_{i}$ and $\beta_{i}$ are the intercept and slope parameters respectively for experiment $i$, and $\varepsilon_{i j} \sim N\left(0, \sigma_{i}^{2}\right)$ is a residual term.

At the second level of the model the slopes $\beta_{i}$ are given a Gaussian Process (GP) prior (Rasmussen and Williams, 2006), such that:

$$
\beta \sim G P(\mu(.), \Sigma(., .))
$$

where $\mu($.$) indicates the mean function, here set to constant \mu_{\beta}$, and $\Sigma(.,$.$) represents the$ autocovariance function. We use an isotropic Gaussian autocovariance such that:

$$
\Sigma_{m n}=\eta_{\beta}^{2} \exp \left[-\rho_{1}\left(V_{m}-V_{n}\right)^{2}-\rho_{2}\left(f_{m}-f_{n}\right)^{2}\right]
$$

where $V_{m}$ and $f_{m}$ are the cutting speed and feed rate for experiment $m$. The additional parameters $\eta_{\beta}^{2}, \rho_{1}$ and $\rho_{2}$ control the degree of smoothing across the $\mathbb{R}^{2}$ field; all are restricted to be positive to ensure positive definiteness of the resulting covariance matrix. A similar restriction requires $\Sigma_{m m}=\eta_{\beta}^{2}+\sigma_{\beta}^{2}$ where $\sigma_{\beta}^{2}$ represents experimental replicate variance. When the values $\rho_{1}$ and $\rho_{2}$ are small then the autocovariance decreases slowly with distance and the field is smooth, and vice versa. The $K \times K$ matrix $\Sigma$ forms a valid covariance matrix for the multivariate Gaussian distribution that is used to fit the model.

The model nuisance parameters $\alpha_{i}$ are also at the second level. They are regularised such that $\alpha_{i} \sim N\left(\mu_{\alpha}, \sigma_{\alpha}^{2}\right)$. This enables further borrowing of strength between experiments. At the lowest level the hyper-parameters $\Theta=\left\{\sigma, \sigma_{\alpha}, \mu_{\alpha}, \mu_{\beta}, \eta_{\beta}, \sigma_{\beta}, \rho_{1}, \rho_{2}\right\}$ are constrained using weakly informative prior distributions. Informative prior distributions are to be preferred (such as the GP prior in the second level), but hyper-parameters for GP models are notoriously difficult to obtain so we follow the weakly-informative convention of Gelman (2006). The specific priors used for the experiment described in this paper are detailed in Section 4.

Taken together we form a posterior distribution:

$$
p(\Theta, \beta \mid L, F) \propto p(F \mid L, \alpha, \beta, \sigma) p\left(\beta \mid \mu_{\beta}, \eta_{\beta}, \sigma_{\beta}, \rho_{1}, \rho_{2}\right) p\left(\alpha \mid \mu_{\alpha}, \sigma_{\alpha}\right) p(\Theta)
$$


After fitting we can estimate the predictive distribution of rates $\beta^{*}$ at new values $V^{*}$ and $f^{*}$ via the GP identity:

$$
\beta^{*} \mid \beta, \Theta \sim N\left(\beta+\Sigma_{\beta}^{*} \Sigma_{\beta}^{-1}\left(\beta-\mu_{\beta}\right), \eta_{\beta}^{2}+\sigma_{\beta}^{2}-\Sigma_{\beta}^{*} \Sigma_{\beta}^{-1}\left(\Sigma_{\beta}^{*}\right)^{T}\right)
$$

where $\left[\Sigma_{\beta}^{*}\right]_{m}=\eta_{\beta}^{2} \exp \left[-\rho_{1}\left(V^{*}-V_{m}\right)^{2}-\rho_{2}\left(f^{*}-f_{m}\right)^{2}\right]$. For a proof see Krzanowski (2000). We take account of parametric uncertainty by integrating over the other parameters:

$$
p\left(\beta^{*} \mid F, L\right)=\int p\left(\beta^{*} \mid \Theta, \beta\right) p(\Theta, \beta \mid L, F) \partial \Theta \partial \beta
$$

where the first term in the integrand is the GP identity and is the second is the posterior distribution.

We fit the model using the probabilistic programming language Stan (Carpenter et al, 2016) through the R package rstan. The package uses Hamiltonian Monte Carlo, a variant on the standard Markov chain Monte Carlo (Brooks et al, 2011), which samples all parameters simultaneously using the derivatives of the posterior up to proportionality. The particular method used by rstan is called the No U-turn Sampler (NUTS) (Hoffman and Gelman, 2014) which avoids over-shooting when sampling parameters in particular directions. In contrast to other HMCMC algorithms, the NUTS algorithm does not require the user to specify the step size or the number of steps and thus requires no hand-tuning.

\section{Results}

Twenty one tests were carried out under various settings for cutting speed and feed rate. The exact control parameters used for the experiments are detailed in the Supplementary Material. In general tool life ranged from $10 \mathrm{~m}$ to $255 \mathrm{~m}$, with Test $7\left(v_{c}=20 \mathrm{~m} / \mathrm{min}, f=\right.$ $45 \mu \mathrm{m} / \mathrm{rev})$ showing the longest tool life $(255 \mathrm{~m})$ and, correspondingly, Test $10\left(v_{c}=58\right.$ $\mathrm{m} / \mathrm{min}, f=22.5 \mu \mathrm{m} / \mathrm{rev}$ ) giving the shortest $(10 \mathrm{~m})$. As would be expected, higher speeds and larger values for feed rate generally lead to shorter tool life. Given workpiece dimensions, most tests required multiple cutting passes before the tool was worn out. Thus the data obtained from the dynamometer included periods where the toll was not in contact with the workpiece. Changepoint analysis using binary segmentation (Killick and Eckley, 2014; Scott and Knott, 1974) was used as a guide to determine the contact phases. These phases were then concatenated before the model described above was fitted.

The regression model with Gaussian process prior for the slope parameters is fitted for each of the three forces. In order to estimate the model in a Bayesian framework, prior distributions must be specified. The following vague prior distributions were used in the analysis of these data:

$$
\begin{aligned}
& -\sigma_{i}^{2} \sim \operatorname{Half-Cauchy}(0,10) \\
& \text { - } \mu_{\alpha} \sim N\left(0,10^{2}\right) \\
& \text { - } \sigma_{\alpha}^{2} \sim \operatorname{Half}-\operatorname{Cauchy}(0,10) \\
& \text { - } \mu_{\beta} \sim N\left(0,10^{2}\right)
\end{aligned}
$$

$$
\begin{aligned}
& -\sigma_{\beta}^{2} \sim \operatorname{Half}-\text { Cauchy }(0,5) \\
& -\eta_{\beta}^{2} \sim \operatorname{Half}-\operatorname{Cauchy}(0,5) \\
& -\rho_{1}^{-1} \sim \operatorname{Half}-\operatorname{Cauchy}(0,5) \\
& -\rho_{2}^{-1} \sim \operatorname{Half}-\operatorname{Cauchy}(0,5)
\end{aligned}
$$

The convergence of the Markov chains was assessed by examining trace plots of the sampled values and the Potential Scale Reduction Factor (Gelman and Rubin, 1992; Brooks and Gelman, 1998).

The parameter estimates were determined by calculating the mean of the HMCMC samples from the posterior of the model parameters given the data. The estimated rates of change 
of force over cutting distance are reported in the Supplementary material for each of the three forces, $F_{f}, F_{t}$ and $F_{p}$, for each of the 21 experiments.

The obtained parameter estimates can be used to estimate the rate of change of feed force for any combination of feed rate and cutting speed (within a neighbourhood of the observed values). This can be done by interpolating between the estimated slope parameters using the posterior predictive distribution on a pre-defined feed rate/cutting speed grid. Slope estimates corresponding to unobserved experimental settings are formed via the multivariate Gaussian conditional distribution of the Gaussian process given the twenty one latent slope estimates. Standard deviations of these estimates can be obtained similarly. More information on Gaussian processes for fitting and prediction can be found in Rasmussen and Williams (2006).

The first columns of Figure 6 shows surface plots for the estimated rates of change in each of the three forces varying by speed and thickness. A grid of 400 points was formed on the illustrated region and the rate of change in force was estimated at each point using the Gaussian process. Standard deviations of these estimates are shown in the surface plots given in the second column.

In a separate analysis, a similar Gaussian process model was used to relate tool life (m) to the two variables of interest, cutting speed and feed rate. This model did not require the top level linear regression step. Once again, the NUTS algorithm was used to fit the model. Figure 7a shows a surface plot illustrating tool life as a function of $v_{c}$ and $f$. Figure $7 \mathrm{~b}$ shows the standard deviations of these estimates.

The response surface plots (Figure 6) map out the rates of change of the force components as a function of the most fundamental cutting parameters. The top left and bottom left panels demonstrate a clear correlation between the rates of change of the tangential $\left(F_{t}\right)$ and feed force $\left(F_{f}\right)$ components, while the mapping of the passive force $F_{p}$ component rate of change is characteristically different. This is attributed physically to the kinematics and the tool geometry in turning where the magnitude of $F_{t}$ and $F_{f}$ are both determined by the removal of most material on the side edge (see Figure 4) of the tool in the feed direction. The corollary is that the nose radius is primarily subjected to lower levels of locally varying undeformed chip thickness with mechanisms of surface plastic deformation and elastic friction applying without material removal (Chou and Song, 2004). Thus the rates of change in $F_{t}$ and $F_{f}$ are expected to be related at least to the measured flank wear levels. This is indeed surmised by the inverse correlation of the tool life as presented in Figure 7. Nonetheless the rates of change in $F_{p}$ are expected to be indicative of other (potentially adverse) effects in turning, for example changes in surface finish and integrity (Pawade et al, 2007).

We conclude that the rates of change in $F_{t}$ and $F_{f}$ correlate with tool-wear rate and provide useful surrogate measures. The response surface plots shown enable the determination of parameter ranges that result in maximum tool life and also prediction by interpolation of results for values not specifically set-up in the experiment (but within the range examined). For example, the use of low cutting speeds and high feed rate will extend tool life while maintaining high material removal rates (higher feed rate decreases the surface roughness but this is not considered here). The results also provide a baseline for comparison of future research on tool-wear in turning and milling of ASTM F75 Co-Cr-Mo and can be used for optimisation of the manufacturing process. This methodology can be further utilised to compare the tool life for different cutting inserts. It should be noted however, that this model should not be used to estimate the rate of change in cutting forces by extrapolating far outside the range of cutting speeds and feed rates considered in this experiment. A further experiment, at low $v_{c}$ and $f$, settings would provide a more detailed description of the profile of the components of the cutting force and progression of the tool wear. 

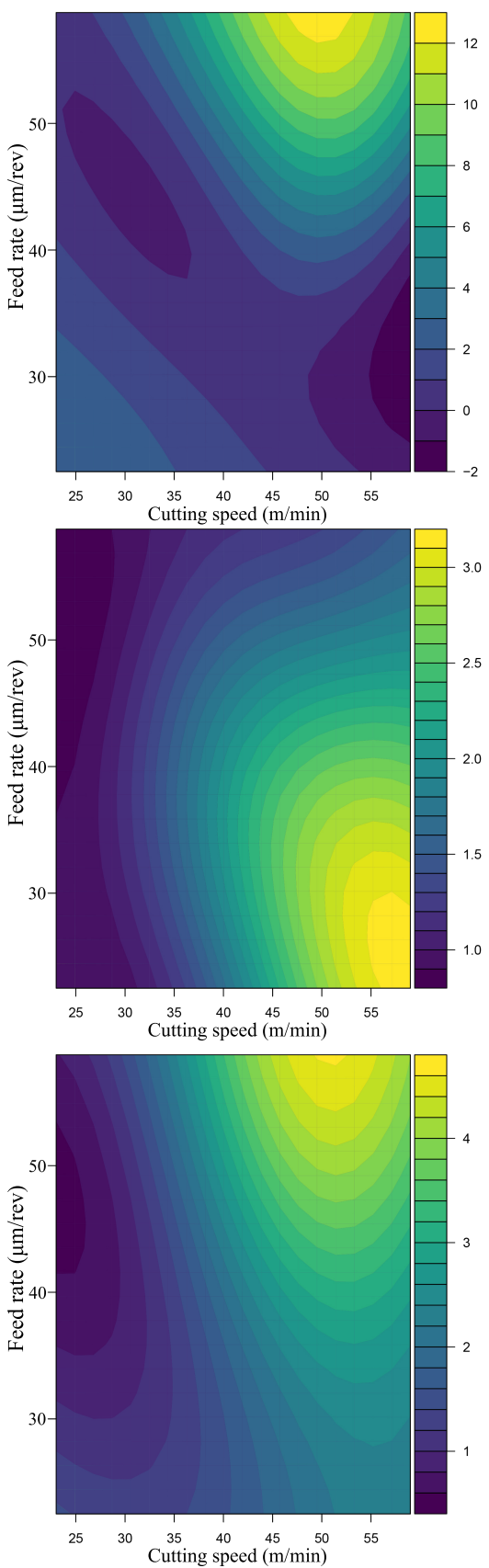
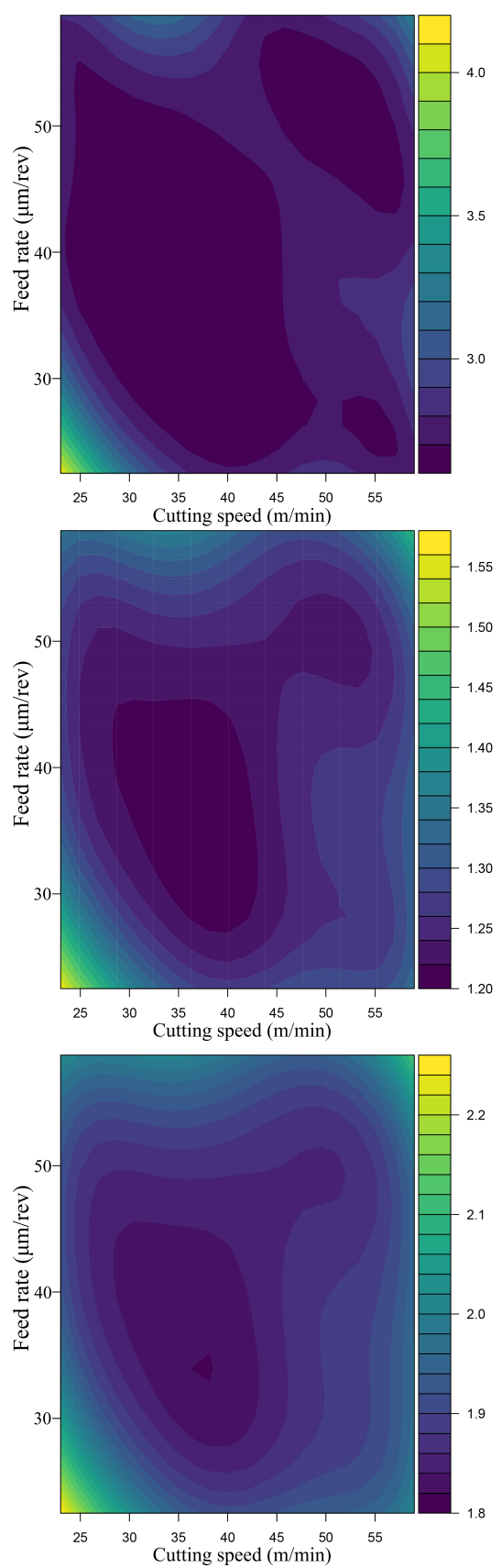

Fig. 6: The first column shows the estimated mean rates of changes whilst the second shows the estimated standard deviations. Note the differing scales. The rows correspond to the rate of change of feed force $\left(F_{f}\right)$, passive force $\left(F_{p}\right)$ and tangential force $\left(F_{t}\right)$ respectively. Each surface is a function of feed rate and cutting speed. 


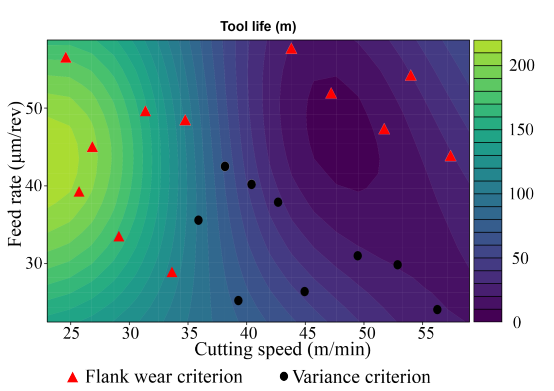

(a)

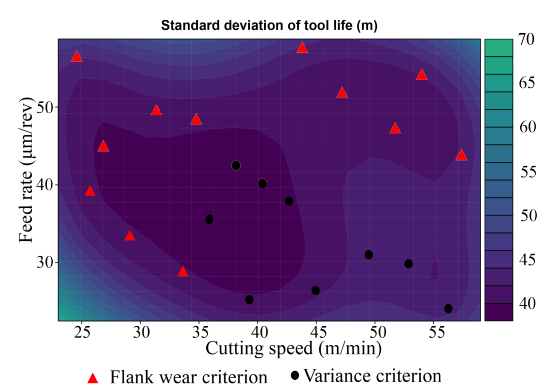

(b)

Fig. 7: The first column shows surface plots indicating the mean tool life (in metres) as a function of feed rate and cutting speed. The second column shows surface plots indicating the standard deviation of these estimates. The tool end-of life criteria are superimposed on both surface plots.

\section{Discussion}

We have presented the results of a novel approach to experimental design applied to the domain of research into machining processes. Specifically, it presents the results and analysis of tool wear progression in turning of medical grade Cobalt Chromium Molybdenum Alloy (ASTM F75). The Sobol sequence design methodology employed allows for the maximum number of tests to be carried out in a designated period of time while also covering the entire parameter range under investigation. We believe it has potential benefits to industry, particularly in time consuming tool-wear trials. While tool wear studies have employed data analytic techniques, to the best of our knowledge the use of Gaussian processes in the manner presented here is novel in this area. The change-point detection technique used allows for filtering out of non-contact and ramp-up stages of the force measurements in this type of experiment and has potential for implementation in indirect, in-process TCM systems.

An examination of the worn cutting tools under scanning electron microscope (SEM) and energy dispersive X-ray spectroscopy (EDX) has indicated the formation of an adhering layer on both the rake and the flank face of the cutting tool and an edge chipping. EDX analysis of this layer has shown a high content of cobalt, chromium and molybdenum. It is most probable that these elements are deposited from the work material in a complex physio-chemical reaction under the extreme conditions pertaining at the tool-workpiece interaction. Moreover, it should be noted that this is an adhering layer and not 'built-up edge'. The adhering layer was more extensive when lower cutting speeds were employed and was usually located $<10 \mu \mathrm{m}$ from the cutting edge. Higher cutting speeds resulted in chipping of large fragments of the cutting edge. This is shown in Figure 8.

The characteristic pattern of the adhered layer is shown in Figure 9. The formation of such a layer was also observed when turning Inconel. However, due to the difference in alloy composition, the mechanisms of formation may be different (Xue and Chen, 2011). As shown in Figure 9, four different zones can be identified. Moving away from the cutting edge, in the direction of the chip flow, the first, a few $\mu m$ wide zone comprise mostly of tungsten and carbon (via EDX analysis). The next zone comprises mostly of cobalt, chromium and molybdenum. It is followed by a wider zone containing tungsten and carbon, therefore it is presumed to be the exposed tungsten-carbide. This is followed by a highly irregular layer 
of adhered cobalt, chromium and molybdenum. This configuration may suggest a sensitivity of this adhering layer to the distribution of the friction/stress/temperature on the rake face of the cutting tool. As the tungsten carbide insert contained a cobalt binder, the mechanism of formation of such layer is presumed due to chemical diffusion/attrition. Specific configuration of the cutting parameters and edge geometry, may form a protective layer and result in flank wear only (Itakura et al, 1999). In future work, we intend to further explore the mechanisms of tool wear and the performance various tool technologies in cutting of Co-Cr-Mo. Any future results will be compared to the baseline results provided in this paper.

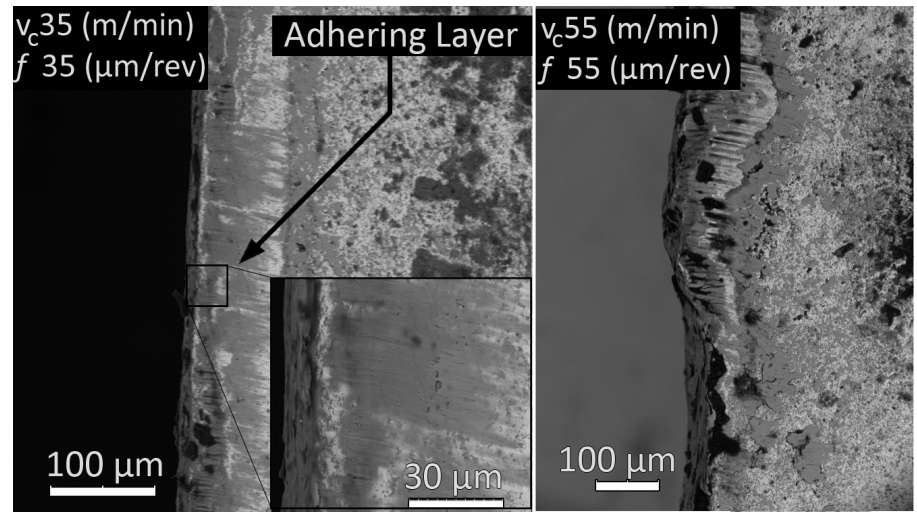

Fig. 8: Comparison of worn cutting edge under SEM

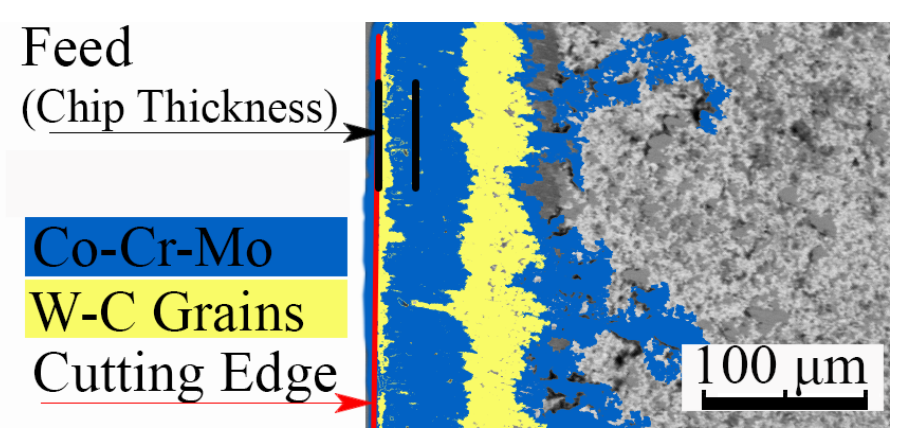

Fig. 9: Map of the adhering layers location on the rake face under SEM

The tool wear results we have presented thus provide a basis for process control and optimisation for given configurations of the manufacturing process. To further this research, context specific analysis should consider the cost of cutting time versus tool life/cost with a view to optimising the overall manufacturing process chain. Another area of further work will apply the techniques developed here to evaluate the performance of different cutting tools. Optimising tool life in this way could produce significant improvements in overall operating effectiveness and gains in many manufacturing contexts. 
Acknowledgements We would like to thank DePuy Synthes and Enterprise Ireland for supporting this research through the Innovation Partnership (IP) programme. The Innovation Partnership programme is cofunded by the European Union through the European Regional Development Fund 2014-2020.

\section{References}

Abouelatta O, Madl J (2001) Surface roughness prediction based on cutting parameters and tool vibrations in turning operations. Journal of materials processing technology 118(1):269-277

Abukhshim N, Mativenga P, Sheikh M (2006) Heat generation and temperature prediction in metal cutting: A review and implications for high speed machining. International Journal of Machine Tools and Manufacture 46(7):782-800

Ahearne E, Baron S, et al (2016) Tool wear in milling of mecical grade cobalt chromium alloy- requirements for advanced process monitoring and data analytics. Proceedings of Machine Tool Technology Research Foundation Annual Meeting San Francisco, California, $5^{\text {th }}-7^{\text {th }}$ July 2016

Al Jabbari Y (2014) Physico-mechanical properties and prosthodontic applications of co-cr dental alloys: a review of the literature. The journal of advanced prosthodontics 6(2):138145

Altintas Y (2012) Manufacturing Automation: Metal Cutting Mechanics, Machine Tool Vibrations, and CNC Design, 2nd edn. Cambridge University Press

ASTM (2016) Standard specification for cobalt-28 chromium-6 molybdenum alloy castings and casting alloy for surgical implants (uns r30075). https: / / www . astm.org/ Standards/F75. htm, accessed: 23/5/2016

Azmin FM, Stobart R (2015) Benefiting from sobol sequences experiment design type for model-based calibration. Tech. rep., SAE Technical Paper

Baron S, Ahearne E (2015) An Investigation of force components in orthogonal cutting of medical grade cobalt chromium alloy (ASTM F1537). CAPE (Computer-Aided Production Engineering) 23: Manufacturing Research and its Applications in the 21st Century

Baron S, Ahearne E, Conolly P, Keaveney S, Byrne G (2015) An assessment of medical grade cobalt chromium alloy ASTM F1537 as a difficult to cut (DTC) material. Proceedings of Machine Tool Technology Research Foundation 2015

Brooks S, Gelman A, Jones G, Meng XL (2011) Handbook of Markov Chain Monte Carlo. CRC press

Brooks SP, Gelman A (1998) General methods for monitoring convergence of iterative simulations. Journal of computational and graphical statistics 7(4):434-455

Buechel Sr FF, Buechel Jr FF, Pappas MJ, D’Alessio J (2001) Twenty-year evaluation of meniscal bearing and rotating platform knee replacements. Clinical Orthopaedics and Related Research 388:41-50

Byrne G, Dornfeld D, Inasaki I, Ketteler G, König W, Teti R (1995) Tool condition monitoring (tcm)the status of research and industrial application. CIRP Annals-Manufacturing Technology 44(2):541-567

Byrne G, Ahearne E, Cotterell M, Mullany B, O?Donnell G, Sammler F (2016) High performance cutting (hpc) in the new era of digital manufacturing-a roadmap. Procedia CIRP 46:1-6

Carpenter B, Gelman A, Hoffman M, Lee D, Goodrich B, Betancourt M, Brubaker MA, Guo J, Li P, Riddell A (2015) Stan: a probabilistic programming language. Journal of Statistical Software 
Carpenter B, Gelman A, Hoffman M, Lee D, Goodrich B, Betancourt M, Brubaker MA, Guo J, Li P, Riddell A (2016) Stan: A probabilistic programming language. J Stat Softw

Chou YK, Song H (2004) Tool nose radius effects on finish hard turning. Journal of Materials Processing Technology 148(2):259-268

Centers for Disease Control, Prevention (2016) Cost of hospital discharges with common hospital operating room procedures in nonfederal community hospitals, by age and selected principal procedure: United states, selected years 2000-2012. http://www . cdc.gov/nchs/data/hus/2014/105.pdf, accessed: 23/5/2016

Dimla D, Lister P (2000) On-line metal cutting tool condition monitoring.: I: force and vibration analyses. International Journal of Machine Tools and Manufacture 40(5):739_ 768

Dudzinski D, Devillez A, Moufki A, Larrouquere D, Zerrouki V, Vigneau J (2004) A review of developments towards dry and high speed machining of inconel 718 alloy. International Journal of Machine Tools and Manufacture 44(4):439-456

Gelman A (2006) Prior distributions for variance parameters in hierarchical models (comment on article by browne and draper). Bayesian Analysis 1(3):515-534

Gelman A, Hill J (2006) Data analysis using regression and multilevel/hierarchical models. Cambridge University Press

Gelman A, Rubin DB (1992) Inference from iterative simulation using multiple sequences. Statistical science pp 457-472

Gelman A, Carlin JB, Stern HS, Rubin DB (2003) Bayesian data analysis, 2nd edn. Chapman \& Hall/CRC Texts in Statistical Science, Chapman and Hall, CRC Press, London, Boca Raton, FL

Girolami M, Calderhead B (2011) Riemann manifold langevin and hamiltonian monte carlo methods. Journal of the Royal Statistical Society: Series B (Statistical Methodology) 73(2):123-214

Halpin T (2012) The wear and failure of pcbn cutting tools in interrupted hard machining. Tech. rep., University College Dublin

Hoffman MD, Gelman A (2014) The no-u-turn sampler: Adaptively setting path lengths in hamiltonian monte carlo. The Journal of Machine Learning Research 15(1):1593-1623

Itakura K, Kuroda M, Omokawa H, Itani H, Yamamoto K, Ariura Y (1999) Wear mechanism of coated cemented carbide tool in cutting of inconel 718 super-heat-resisting alloy. International Journal of the Japan Society for Precision Engineering 33(4):326-332

Jaspers S, Dautzenberg J (2002) Material behaviour in metal cutting: strains, strain rates and temperatures in chip formation. Journal of materials processing technology 121(1):123135

Kaiser R (2013) Study of the effect of casting variables and heat treatment procedure on the microstructure and mechanical properties of co-cr-mo biomedical alloy. Tech. rep., University College Dublin

Killick R, Eckley I (2014) changepoint: An r package for changepoint analysis. Journal of Statistical Software 58(3):1-19

Kistler (2016) Multicomponent dynamometer technical specification sheet. https: // www.kistler.com/ ? type $=669$ \& fid=41281, accessed: 23/5/2016

Klocke F, Krieg T (1999) Coated tools for metal cutting-features and applications. CIRP Annals-Manufacturing Technology 48(2):515-525

Krzanowski W (2000) Principles of multivariate analysis. OUP Oxford

Markopoulos AP (2013) Finite Element Method in Machining Processes, Springer London, London, chap Cutting Mechanics and Analytical Modeling, pp 11-27 
Merchant ME (1945) Mechanics of the metal cutting process. i. orthogonal cutting and a type 2 chip. Journal of Applied Physics 16(5):267-275

Neal RM, et al (2011) Mcmc using hamiltonian dynamics. Handbook of Markov Chain Monte Carlo 2:113-162

Pawade R, Joshi SS, Brahmankar P, Rahman M (2007) An investigation of cutting forces and surface damage in high-speed turning of inconel 718. Journal of Materials Processing Technology 192:139-146

Rasmussen C, Williams K (2006) Principles of multivariate analysis. MIT Press

Scott AJ, Knott M (1974) A cluster analysis method for grouping means in the analysis of variance. Biometrics pp 507-512

Settineri L, Faga MG, Lerga B (2008) Properties and performances of innovative coated tools for turning inconel. International Journal of Machine Tools and Manufacture 48(7):815-823

Shaw MC (2005) Metal Cutting Principles, 2nd edn. Oxford University Press, New York

Sobol IM (1967) On the distribution of points in a cube and the approximate evaluation of integrals. Zhurnal Vychislitel'noi Matematiki i Matematicheskoi Fiziki 7(4):784-802

Teti R, Jemielniak K, ODonnell G, Dornfeld D (2010) Advanced monitoring of machining operations. CIRP Annals-Manufacturing Technology 59(2):717-739

Tozzi J (2015) Older, heavier americans fuel a $\$ 4$ billion knee replacement market. http://www.bloomberg.com/news/articles/2015-09-02/ older-heavier-americans-fuel-a-4-billion-knee-replacement-market, accessed: 23/5/2016

Uhlmann E, Fuentes JO, Keunecke M (2009) Machining of high performance workpiece materials with cbn coated cutting tools. Thin solid films 518(5):1451-1454

Williams D (2008) On the mechanisms of biocompatibility. Biomaterials 29(20):2941-2953

Xue C, Chen W (2011) Adhering layer formation and its effect on the wear of coated carbide tools during turning of a nickel-based alloy. Wear 270(11-12):895-902 